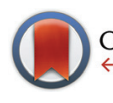

CrossMark \&lick for updates

Cite this: Dalton Trans., 2015, 44 10673

Received 4th December 2014 Accepted 19th January 2015

DOI: $10.1039 / c 4 d t 03721$ a

www.rsc.org/dalton

\title{
Structural and dielectric studies of the phase behaviour of the topological ferroelectric $\mathrm{La}_{1-x} \mathrm{Nd}_{x} \mathrm{TaO}_{4} \dagger$
}

\author{
Keith J. Cordrey, ${ }^{a}$ Magda Stanczyk, ${ }^{a}$ Charlotte A. L. Dixon, ${ }^{a}$ Kevin S. Knight, ${ }^{\text {b }}$ \\ Jonathan Gardner, ${ }^{a}$ Finlay D. Morrison ${ }^{a}$ and Philip Lightfoot*a
}

\begin{abstract}
The layered perovskite $\mathrm{LaTaO}_{4}$ has been prepared in its polar orthorhombic polymorphic form at ambient temperature. Although no structural phase transition is observed in the temperature interval $25^{\circ} \mathrm{C}<T<$ $500{ }^{\circ} \mathrm{C}$, a very large axial thermal contraction effect is seen, which can be ascribed to an anomalous buckling of the perovskite octahedral layer. The non-polar monoclinic polymorph can be stabilised at ambient temperature by $\mathrm{Nd}$-doping. A composition $\mathrm{La}_{0.90} \mathrm{Nd}_{0.10} \mathrm{TaO}_{4}$ shows a first-order monoclinicorthorhombic (non-polar to polar) transition in the region $250^{\circ} \mathrm{C}<T<350^{\circ} \mathrm{C}$. Dielectric responses are observed at both the above structural events but, despite the 'topological ferroelectric' nature of orthorhombic $\mathrm{LaTaO}_{4}$, we have not succeeded in obtaining ferroelectric $P-E$ hysteresis behaviour. Structural relationships in the wider family of $A_{n} B_{n} X_{3 n+2}$ layered perovskites are discussed.
\end{abstract}

\section{Introduction}

The mechanism of the paraelectric-ferroelectric phase transition in most well-studied oxide ferroelectrics lies in the condensation of a polar soft-phonon mode, typically driven by offcentring of an octahedral ' $\mathrm{d}$ cation' or a 'lone-pair cation' via the $2^{\text {nd }}$-order Jahn-Teller effect. ${ }^{1}$ This is called proper ferroelectricity, and examples are the classic perovskite ferroelectrics $\mathrm{BaTiO}_{3}, \mathrm{PbTiO}_{3}$ and $\mathrm{BiFeO}_{3}$. However, in recent years several new mechanisms that drive ferroelectricity have been discovered or postulated. These include so-called 'geometric ferroelectricity', first proposed theoretically for hexagonal $\mathrm{YMnO}_{3}{ }^{2,3}$ and later confirmed experimentally, ${ }^{4}$ and 'hybrid improper ferroelectricity' (HIF), ${ }^{5}$ which requires coupling of two distinct non-polar modes to produce a net polar symmetry. These latter two mechanisms are both types of improper ferroelectricity, where the polar modes arise as a natural 'sideeffect' from strictly non-polar lattice modes. In turn these nonpolar modes are generally driven by purely geometric (e.g., ionic size) effects: tilting of $\mathrm{MnO}_{5}$ polyhedra in the case of $\mathrm{YMnO}_{3}$, and octahedral rotations and/or cation ordering in the more general case of HIF. Significantly, the non-polar modes

\footnotetext{
${ }^{a}$ EaStCHEM and School of Chemistry, University of St Andrews, St Andrews, Fife KY16 9ST, UK. E-mail: pl@st-and.ac.uk

${ }^{b}$ ISIS Facility, STFC Rutherford Appleton Laboratory, Harwell, Oxfordshire OX11 $O Q X, U K$

$\dagger$ Electronic supplementary information (ESI) available. See DOI: 10.1039/ c4dt03721a
}

are the primary order parameters driving the phase transition, which then permit the polar modes as secondary order parameters. More recently, ${ }^{6}$ it has been observed that a purely 'geometric' effect can also operate to produce a proper ferroelectric; i.e., the lattice distortion is not driven by one of the two common 'electronic' factors above, but simply by a structural (size) mismatch of some sort. In this case, however, this geometric effect directly gives rise not only to a polar space group, but to the polarisation itself, without the need for a 'slave' polar mode. It has now been suggested that the name 'topological ferroelectricity' rather than geometric ferroelectricity be applied to this type of mechanism. ${ }^{7}$ Note that use of the term 'ferroelectric' in these definitions refers to an intrinsic structural polarity, which is in principle switchable, and does not necessarily imply experimental observation of switchability. The mechanism has been proposed within the class of layered perovskites of generic formula $\mathrm{A}_{n} \mathrm{~B}_{n} \mathrm{X}_{3 n+2},{ }^{8}$ specifically for the cases $\mathrm{BaMnF}_{4}{ }^{6}(n=2)$ and $\mathrm{La}_{2} \mathrm{Ti}_{2} \mathrm{O}_{7}{ }^{7}(n=4)$. The key point is that the layered nature of the perovskite topology means that rigid octahedral tilting, ubiquitous in perovskite crystallography, can naturally give rise to a polarisation since the number of 'oxygen planes' is odd (Fig. 1); this contrasts with the case in three-dimensionally connected perovskites, where octahedral tilt modes are intrinsically centrosymmetric.

In this paper we explore some of the crystal chemistry of another member of the $\mathrm{A}_{n} \mathrm{~B}_{n} \mathrm{X}_{3 n+2}$ family, viz. $\mathrm{LaTaO}_{4}$. This compound can exist in two polymorphic forms when prepared by 'traditional' high-temperature solid-state routes ${ }^{9-12}$ and a third polymorph can also be prepared via decomposition of a 

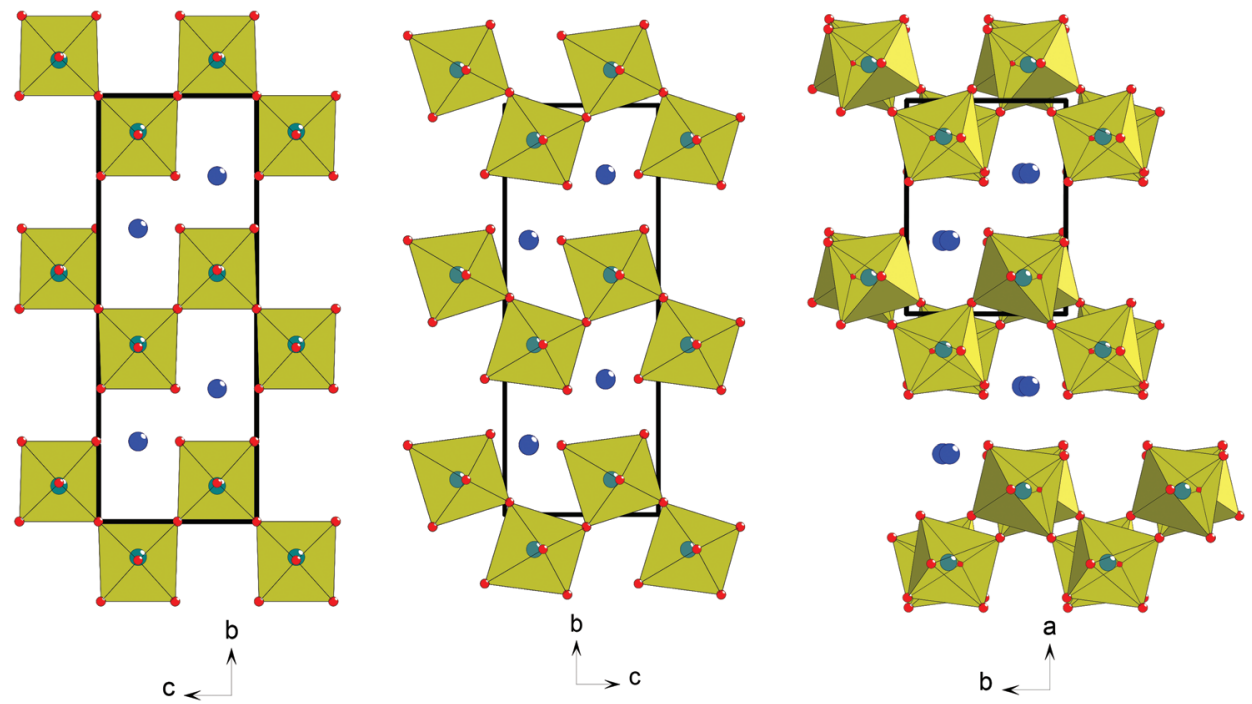

Fig. 1 (a) Aristotype $\mathrm{ABX}_{4}$ structure type, space group $\mathrm{Cmcm}$, with no octahedral tilting. (b) O-LaTaO ${ }_{4}$ structure, space group $\mathrm{Cmc}_{1}$ : note that the $c$-axis polarity arises as a natural consequence of the 'in-phase' tilting around the $a$-axis. (c) $M-\mathrm{LaTaO}_{4}$ structure, space group $P 2_{1} / c$ : in this case the 'anti-phase' tilting around $c$ permits retention of centrosymmetricity.

hydrated precursor. ${ }^{13}$ Of the former two phases, one is orthorhombic and polar and the other monoclinic and centrosymmetric (hereafter designated $\mathrm{O}-\mathrm{LaTaO}_{4}$ and $\mathrm{M}-\mathrm{LaTaO}_{4}$, respectively, Fig. 1). Earlier studies ${ }^{9-12}$ suggest ${\mathrm{M}-\mathrm{LaTaO}_{4}}_{4}$ is the most stable form at ambient temperature, and that this phase undergoes a phase transition to $\mathrm{O}-\mathrm{LaTaO}_{4}$ at $\sim 175{ }^{\circ} \mathrm{C}^{9}$ or $240{ }^{\circ} \mathrm{C} .{ }^{10}$ In our study we have successfully prepared a nearpure sample of $\mathrm{O}-\mathrm{LaTaO}_{4}$ and we study the structural behaviour of this system versus temperature using high-resolution powder neutron diffraction. We also show that the $\mathrm{M}-\mathrm{LaTaO}_{4}$ phase can be stabilised further at ambient temperature by Nddoping, and study the thermal evolution of this phase, and its transformation into the polar $\mathrm{O}-\mathrm{LaTaO}_{4}$ polymorph. Finally, we carry out some general structural comparisons of these phases and related ones, which may be of relevance to their physical properties.

\section{Experimental}

\section{Synthesis}

Several different procedures were trialled in order to produce phase-pure samples. Typically, stoichiometric ratios of dried component oxides $\left(\mathrm{La}_{2} \mathrm{O}_{3}, \mathrm{Ta}_{2} \mathrm{O}_{5}\right.$ and $\mathrm{Nd}_{2} \mathrm{O}_{3}$; all $\left.>99 \%\right)$ were mixed, either by ball-milling at $600 \mathrm{rpm}$ for 1 hour or manually in a pestle and mortar, before being pressed into a pellet and annealed at temperatures between $1200{ }^{\circ} \mathrm{C}$ and $1400{ }^{\circ} \mathrm{C}$ for 12 hours. For $\mathrm{LaTaO}_{4}$ all these procedures produced phase mixtures of either orthorhombic and monoclinic polymorphs of $\mathrm{LaTaO}_{4}$, or orthorhombic plus a small amount of an unidentified impurity. The highest phase fraction of $\mathrm{LaTaO}_{4}$ (as the $\mathrm{O}-\mathrm{LaTaO}_{4}$ polymorph) was produced in a two-stage process: annealing at $1250{ }^{\circ} \mathrm{C}$ for 6 hours, followed by ballmilling, re-pelleting and annealing for a further 6 hours at
$1450{ }^{\circ} \mathrm{C}$. For the Nd-doped samples, $\mathrm{La}_{1-x} \mathrm{Nd}_{x} \mathrm{TaO}_{4} 0.05<x<$ 0.60 , starting mixtures were hand-ground, pelleted and annealed at $1200{ }^{\circ} \mathrm{C}$ for 6 hours, then re-ground, pelleted and annealed at $1350{ }^{\circ} \mathrm{C}$ for 12 hours. In each case, the best samples were reacted on $\mathrm{Pt}$ foil in open alumina crucibles. For both the O-LaTaO ${ }_{4}$ and $\mathrm{La}_{0.9} \mathrm{Nd}_{0.1} \mathrm{TaO}_{4}$ samples used for electrical characterisation a heating rate of $10{ }^{\circ} \mathrm{C} \min ^{-1}$ was employed and at the end of the heating stage, the furnace was switched off and samples allowed to cool inside the furnace.

\section{Powder diffraction}

Preliminary phase purity and structure analysis for each material was confirmed by Rietveld refinement of X-ray powder diffraction (XPD) data collected on a PANalytical Empyrean X-ray diffractometer using $\mathrm{Cu}-\mathrm{K}_{\alpha 1}$ radiation.

Variable temperature neutron powder diffraction (NPD) data on $\mathrm{LaTaO}_{4}$ and $\mathrm{La}_{0.9} \mathrm{Nd}_{0.1} \mathrm{TaO}_{4}$ were collected on the highresolution instrument (HRPD) at ISIS. For $\mathrm{LaTaO}_{4}$ data were collected at ambient temperature and between $100{ }^{\circ} \mathrm{C}$ and $500{ }^{\circ} \mathrm{C}$ at $50{ }^{\circ} \mathrm{C}$ intervals; for $\mathrm{La}_{0.9} \mathrm{Nd}_{0.1} \mathrm{TaO}_{4}$ date were collected between $100{ }^{\circ} \mathrm{C}$ and $600{ }^{\circ} \mathrm{C}$ at $50{ }^{\circ} \mathrm{C}$ intervals. In each case, data collection times were approximately 40 minutes for $\sim 5 \mathrm{~g}$ samples. For $\mathrm{LaTaO}_{4}$, a preliminary dataset was collected at $25^{\circ} \mathrm{C}$ on the GEM diffractometer.

All quantitative data analysis was carried out by Rietveld refinement using the GSAS program ${ }^{14}$ and its EXPGUI user interface. For each of the HRPD runs, detector banks at $2 \theta \sim$ $168^{\circ}$ and $90^{\circ}$ were analysed simultaneously.

\section{Electrical properties}

Electrodes were fabricated on opposing faces of pelleted samples using either Ag paint or sputtered gold. Dielectric properties were measured from room temperature to $600{ }^{\circ} \mathrm{C}$ using either an Agilent 4294A or Wayne Kerr 6500B impedance ana- 
lyser with $500 \mathrm{mV}$ excitation and heating/cooling rates of $2 \mathrm{~K}$ $\mathrm{min}^{-1}$. Polarisation-field data were obtained using an aixACCT TF2000 analyzer with a TREK $4 \mathrm{kV}$ voltage amplifier.

\section{Results and discussion}

\section{$\mathrm{LaTaO}_{4}$}

All attempted preparations resulted in phase mixtures of either O-LaTaO ${ }_{4}$ and $\mathrm{M}-\mathrm{LaTaO}_{4}$ or O-LaTaO ${ }_{4}$ plus a small amount of unidentified impurity, as evidenced by XPD (see ESI $\dagger$ for standard XPD patterns). Comparing to previous studies, it is not entirely clear why previous authors have isolated predominantly the $\mathrm{M}-\mathrm{LaTaO}_{4}$ phase from apparently similar reactions; for example Cava ${ }^{9}$ used a much higher reaction temperature $\left(1750^{\circ} \mathrm{C}\right)$, whereas Siqueira $^{12}$ used a lower temperature $\left(1300{ }^{\circ} \mathrm{C}\right)$. It is possible that the present ball-milling method (not used in previous studies) is critical, although sample cooling rate is another parameter that has not been quoted or studied in detail in any of the studies so far. The sample with the highest phase fraction of $\mathrm{O}-\mathrm{LaTaO}_{4}$ (and negligible M-LaTaO ${ }_{4}$ ) was chosen for further study by NPD. A typical Rietveld fit is shown in Fig. 2. This refinement is based on a single phase fit to $\mathrm{O}-\mathrm{LaTaO}_{4}$, space group $C m c 2_{1}$, with $a \sim 3.94$, $b \sim 14.7, c \sim 5.6 \AA$ (Fig. 1(b)). Further details of the refinement model and outcomes are given in ESI. $\dagger$ No phase changes were detected throughout the temperature range studied, and the quality of fit to the $\mathrm{O}-\mathrm{LaTaO}_{4}$ model remained similar from $100{ }^{\circ} \mathrm{C}$ to $500{ }^{\circ} \mathrm{C}$. However, an unusual trend in lattice parameters versus temperature was observed. Fig. 3 shows the thermal evolution of unit cell $a, b, c$, and $V$ parameters. Of particular note is the very large negative expansivity of the $b$-axis $\left(\alpha_{b} \sim-40 \times 10^{-6} \mathrm{~K}^{-1}\right)$ in the temperature range $100-300{ }^{\circ} \mathrm{C}$, leading to a near-zero volume expansivity around $200-250{ }^{\circ} \mathrm{C}$.

The crystal structure (Fig. 1(b), 4) consists of perovskite-like blocks of corner-shared octahedra extending in the ac plane, separated by the $\mathrm{La}^{3+}$ cations, which may be regarded as ninecoordinated to oxide. In order to pinpoint the structural features leading to the anomalous expansivity behaviour, we can

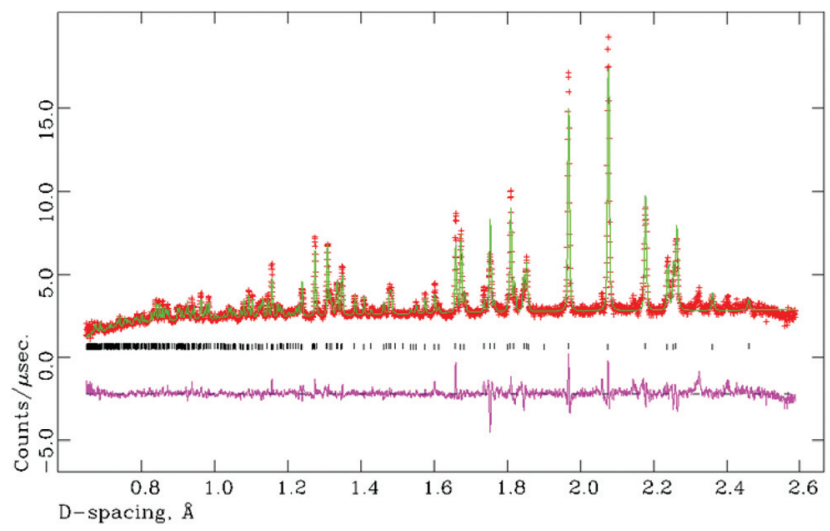

Fig. 2 Rietveld fit (NPD) for single phase $\mathrm{O}-\mathrm{LaTaO}_{4}$ model at $100{ }^{\circ} \mathrm{C}$. Overall $\chi^{2}=6.67$ for 37 refined variables. define three parameters within these two distinct blocks: the thickness of the inter-layer block, $d 1$, the thickness of the perovskite block, $d 2$, and the angle of tilt within the corrugated octahedral layers, $\omega$. From the geometry of these definitions it can be seen that

$$
b=2(d 1+d 2)
$$

and also that the tilt angle, $\omega$, will have a significant effect on $d 2$. Thermal evolution of these three parameters is shown in Fig. 5. As can be seen, the variation of the $d 1$ parameter is insignificant over the temperature range studied. However, $\omega$, and correspondingly $d 2$, shows a significant change: contraction across the region $100-300{ }^{\circ} \mathrm{C}$, followed by a plateau. We conclude that the structural effect driving the anomalous expansivity of the $b$-axis is this 'buckling' of the perovskite layer allowed by the flexibility of the inter-octahedral angle, $\omega$, and perhaps driven by the differing changes in bonding requirements of the La and Ta sites (ESI $\dagger$ ) versus temperature.

A recent variable temperature $(90-350 \mathrm{~K})$ structural study of some higher $n$ members of this family, $\mathrm{La}_{n}(\mathrm{Ti}, \mathrm{Fe})_{n} \mathrm{O}_{3 n+2}(n=5$ and 6$)^{15}$ showed that in those cases the thermal variation in the interlayer distance parameter is also insignificant, whereas the thickness of the perovskite block showed a marked increase, with no observable anomalies.

$\mathrm{La}_{1-x} \mathrm{Nd}_{x} \mathrm{TaO}_{4}(0.05<x<0.60)$

The motivation for preparing this series was to stabilise the M-LaTaO ${ }_{4}$ structure rather than $\mathrm{O}-\mathrm{LaTaO}_{4}$ phase at ambient temperature. This postulate was based on the previous observation by Cava ${ }^{9}$ that the $\mathrm{M}-\mathrm{LnTaO}_{4}$ to $\mathrm{O}-\mathrm{LnTaO}_{4}$ phase transition temperature increases rapidly with decreasing ionic size of $\operatorname{Ln}^{3+}$ : $\mathrm{La}\left(175^{\circ} \mathrm{C}\right)<\mathrm{Ce}\left(818^{\circ} \mathrm{C}\right)<\operatorname{Pr}\left(1300^{\circ} \mathrm{C}\right)$. These are the only three lanthanides that adopt either of these structure types under ambient pressure, although we note that pure $\mathrm{M}-\mathrm{NdTaO}_{4}$ can be prepared from the fergusonite polymorph under extreme conditions ( $\left.8 \mathrm{MPa}, 1500{ }^{\circ} \mathrm{C}\right) .{ }^{16}$ As the change in stability of the M- versus O-polymorphs appears to be so dramatically influenced by small changes in ionic size, it was anticipated that a small amount of $\mathrm{Nd}^{3+}$-doping into O-LaTaO would trigger a transformation to the $\mathrm{M}$-phase; this was indeed found to be the case.

For values of $0.05 \leq x \leq 0.40$, preliminary analysis of XPD data suggested that the $\mathrm{M}-\mathrm{LaTaO}_{4}$ could be prepared almost phase-pure. For $x=0.50$ and 0.60 a significant additional phase (fergusonite type $\mathrm{LnTaO}_{4}$ ) was observed. Rietveld analysis of this series confirmed the extent of the $\mathrm{M}-\mathrm{LaTaO}_{4}$ solid solution in $\mathrm{La}_{1-x} \mathrm{Nd}_{x} \mathrm{TaO}_{4}$ as $0.05 \leq x \leq 0.40$. Lattice parameters across the solid solution vary monotonically with $x$; these were determined by using a model with fixed atomic coordinates. An example Rietveld plot is given in Fig. 6, and further details are given in the ESI. $\dagger$

A sample of $\mathrm{M}-\mathrm{La}_{0.9} \mathrm{Nd}_{0.1} \mathrm{TaO}_{4}$ was studied versus temperature by NPD, up to $600{ }^{\circ} \mathrm{C}$. As anticipated, a phase transition from the $\mathrm{M}$ - to the $\mathrm{O}$-phase was observed within the temperature range studied. This transition is of a first-order nature, as shown by the co-existence of both phases with the region 

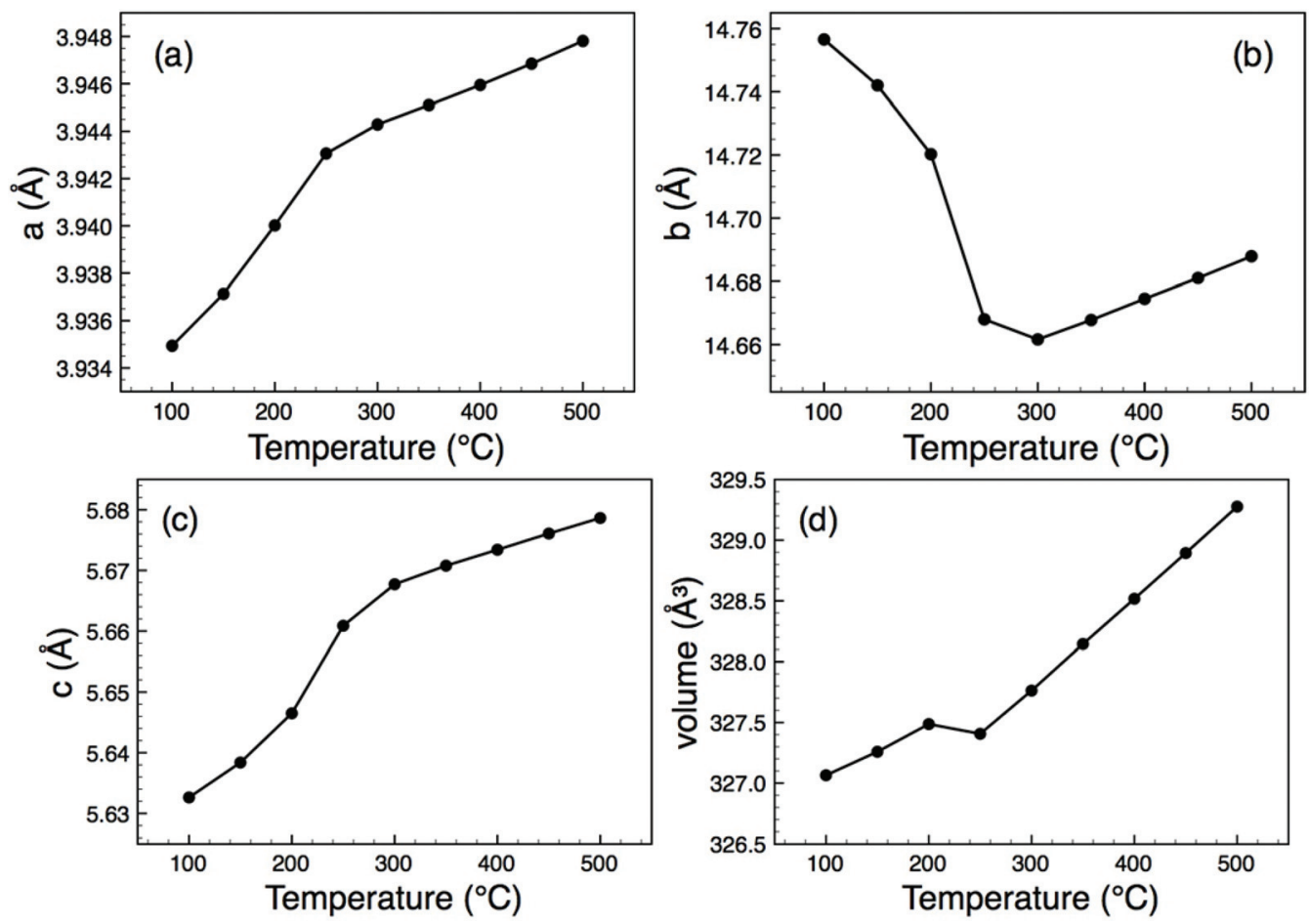

Fig. 3 Thermal evolution of lattice parameters and unit cell volume for O- $\mathrm{LaTaO}_{4}$, derived from Rietveld refinement of NPD data.

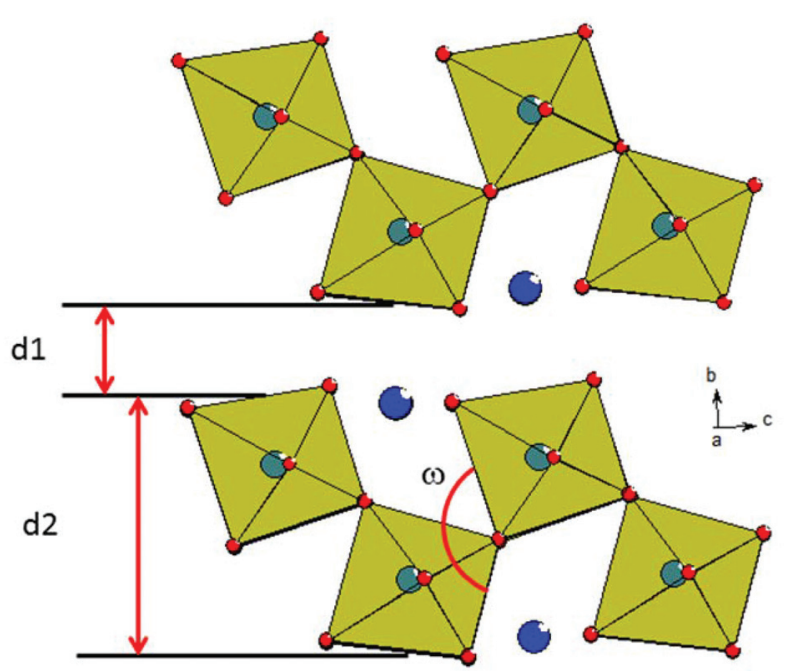

Fig. 4 View of adjacent octahedral blocks in the O- $\mathrm{LaTaO}_{4}$ structure, with the definitions of the parameters, $d 1, d 2$ and $\omega$ shown.

$250<T<350{ }^{\circ} \mathrm{C}\left(\mathrm{M} / \mathrm{O}\right.$ phase fractions (\%) at $250{ }^{\circ} \mathrm{C}, 300{ }^{\circ} \mathrm{C}$ and $350{ }^{\circ} \mathrm{C}$ are $90 / 10,59 / 41$ and $24 / 76$, respectively). In fact, there is no simple group-subgroup relationship between the two crystal structures, so the transition is expected to be $1^{\text {st }}$ order according to Landau theory. Rietveld fits are shown in Fig. 7(a) and (b) for single-phase fits to the M- and O-models at 100 and $500{ }^{\circ} \mathrm{C}$, respectively. Attempts to refine the hightemperature phase in the centrosymmetric parent phase, space group $\mathrm{Cmcm}$ (Fig. 1(a)), led to significantly poorer fits and unrealistic atomic displacement parameters $\left(C m c 2_{1}\right.$ model: 37 variables, $\chi^{2}=3.26, \mathrm{Cmcm}$ model: 30 variables, $\left.\chi^{2}=13.5\right) . \mathrm{A}$ similar contraction of the $b$-axis, as observed for the O-LaTaO phase, is seen here in the region $250<T<450{ }^{\circ} \mathrm{C}$. Further details are given in ESI. $\dagger$

\section{Electrical properties}

Dielectric data for $\mathrm{O}-\mathrm{LaTaO}_{4}$ and $\mathrm{M}-\mathrm{La}_{0.9} \mathrm{Nd}_{0.1} \mathrm{TaO}_{4}$ are shown in Fig. 8 (additional plots at a range of frequencies are provided in ESI $\dagger$ ). It should be noted that the presence of other phase(s) in these samples are at level unlikely to contribute significantly to the overall dielectric response given the low volume fraction and similarity in relative permittivity. O-LaTaO ${ }_{4}$ exhibits a broad peak in permittivity with a maximum at $c a .200{ }^{\circ} \mathrm{C}$, Fig. 8(a), which corresponds to the structural change observed in the diffraction data. The peak is observed on both heating and cooling, indicating this structural relaxation is reversible and shows no thermal hysteresis. Data for $\mathrm{M}-\mathrm{La}_{0.9} \mathrm{Nd}_{0.1} \mathrm{TaO}_{4}$ show a sharp increase in permittivity beginning at $c a .250{ }^{\circ} \mathrm{C}$ on heating, corresponding to the M-O phase transition, Fig. 8(b). This feature shows a large thermal hysteresis consistent with the first order nature of the phase transition. A second feature in the permittivity in the form of a small peak at $\mathrm{ca} .400{ }^{\circ} \mathrm{C}$ is evident on both heating and cooling (and with no temperature hysteresis). The overall dielectric response for this composition is reminiscent of that observed for the ferroelectric tetragonal tungsten bronze compound $\mathrm{GdK}_{2} \mathrm{Nb}_{5} \mathrm{O}_{15}$ recently reported by Gagou et al. ${ }^{17}$ based on diffraction data the authors of that study assigned the small peak in permittivity to an antiferroelectric-paraelectric phase transition. Unfortunately we have insufficient high 

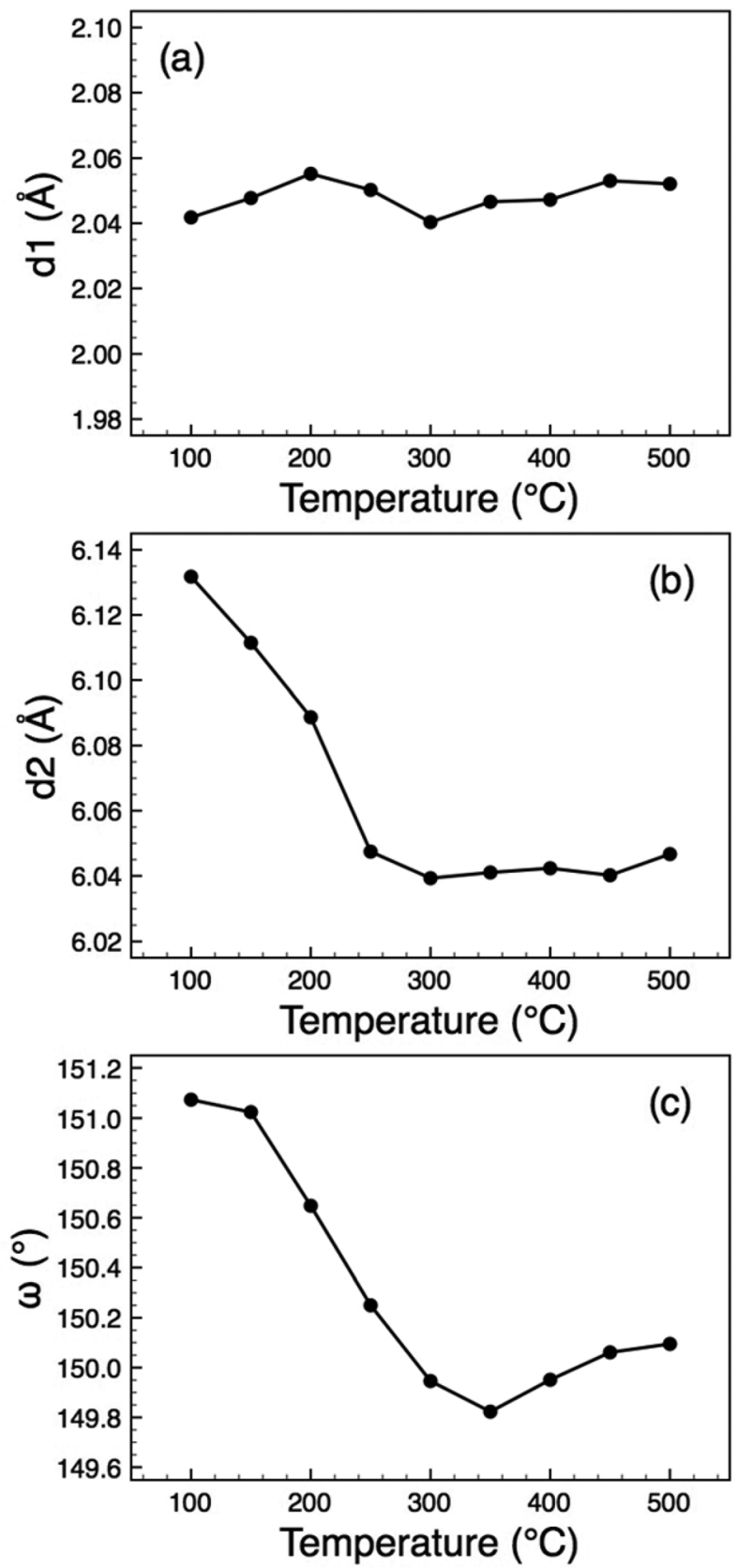

Fig. 5 Thermal evolution of the distortion parameters (defined in Fig. 4) for $\mathrm{O}-\mathrm{LaTaO}_{4}$, (a) $d 1$, (b) $d 2$, (c) $\omega$.

temperature diffraction data for our $\mathrm{La}_{0.9} \mathrm{Nd}_{0.1} \mathrm{TaO}_{4}$ sample in this region to be able to make a similar comparison, but it is worthy to note that short range antiferroelectric ordering has been reported in isostructural $\mathrm{BaMnF}_{4},{ }^{18}$ which arises from an alternate rotation direction of perovskite blocks along the $a$-axis giving an incommensurate modulation. Also, if the "anti-polar" alternate octahedral rotations have no long-range order but are present as nano-domains or interspersed in a manner akin to stacking faults, these are unlikely to be readily detectable by diffraction.

Polarisation-field $(P-E)$ measurements were carried out on $\mathrm{O}-\mathrm{LaTaO}_{4}$ in an attempt to confirm the ferroelectric nature of

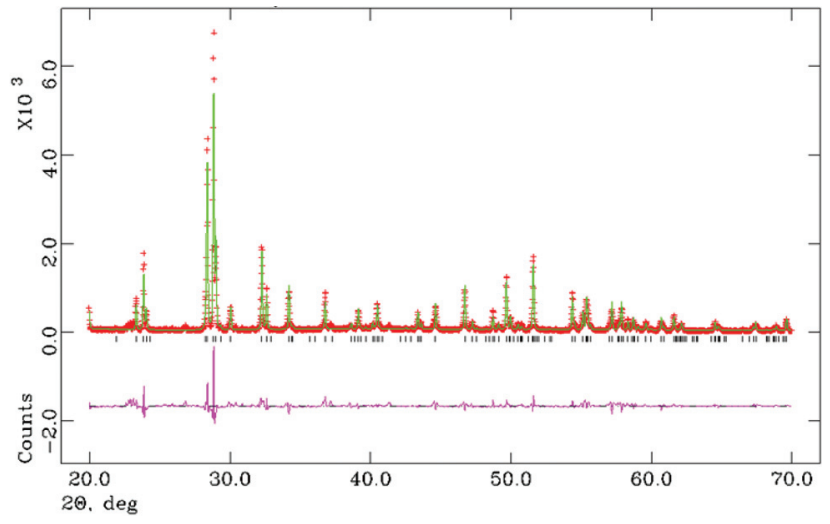

Fig. 6 Rietveld fit (XPD) for $\mathrm{La}_{0.80} \mathrm{Nd}_{0.20} \mathrm{TaO}_{4}\left(\mathrm{M}-\mathrm{LaTaO}_{4}\right)$ model at $25^{\circ} \mathrm{C} \cdot \chi^{2}=4.91$.
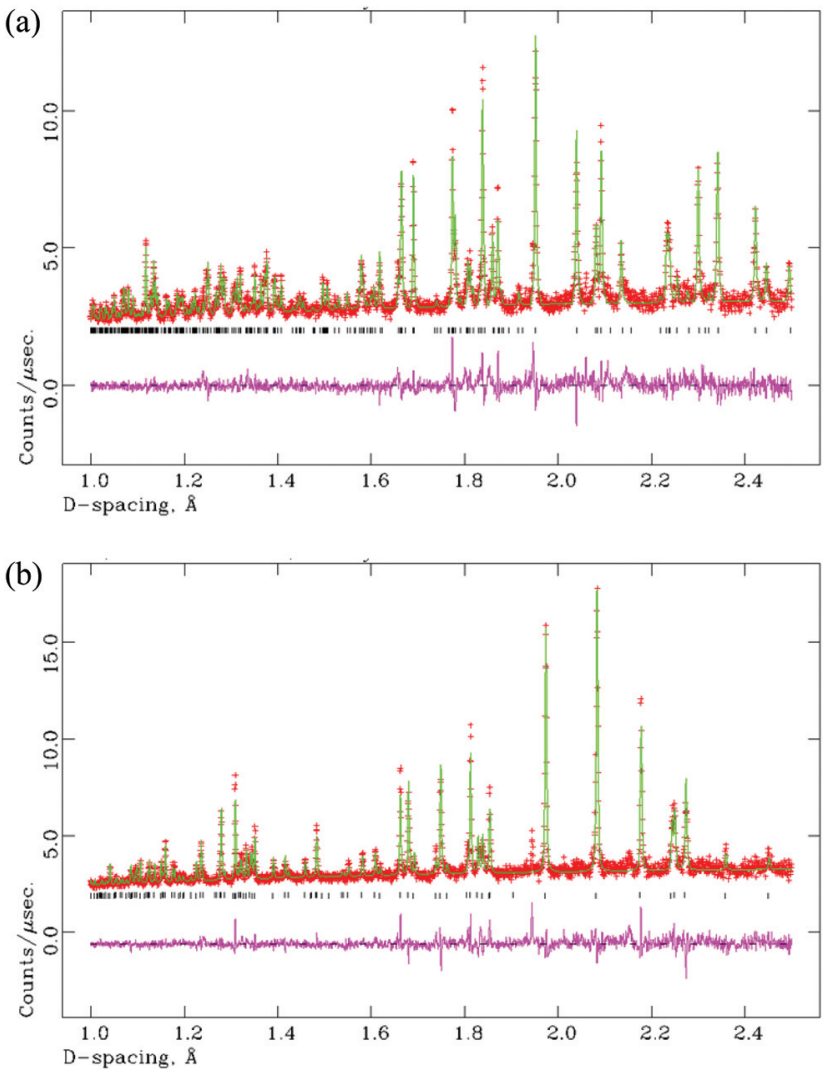

Fig. 7 Rietveld fits (NPD) for $\mathrm{La}_{0.90} \mathrm{Nd}_{0.10} \mathrm{TaO}_{4}$ at (a) $100{ }^{\circ} \mathrm{C}\left(\mathrm{M}-\mathrm{LaTaO}_{4}\right.$ model); $\chi^{2}=2.58$ for 45 variables, and (b) $500{ }^{\circ} \mathrm{C}\left(\mathrm{O}-\mathrm{LaTaO}_{4}\right.$ model); $\chi^{2}=3.26$ for 37 variables. Note that the prominent peak at $d \sim 1.95 \AA$ is instrumental (it does not appear in the room-temperature XPD data).

this polymorph. $P-E$ data obtained at room temperature under an applied field of $100 \mathrm{kV} \mathrm{cm}^{-1}$ at $1 \mathrm{kHz}$ showed a linear dielectric response and no evidence of ferroelectric switching (see $\mathrm{ESI}^{\dagger}$ ). The coercive field for isostructural $\mathrm{BaMgF}_{4}$ is known to increase dramatically with frequency, ${ }^{19,20}$ suggesting a high nucleation energy for switching. In order to investigate this, measurements were repeated at lower frequencies (down to $0.1 \mathrm{~Hz}$ ). Unfortunately, however, under these conditions the 

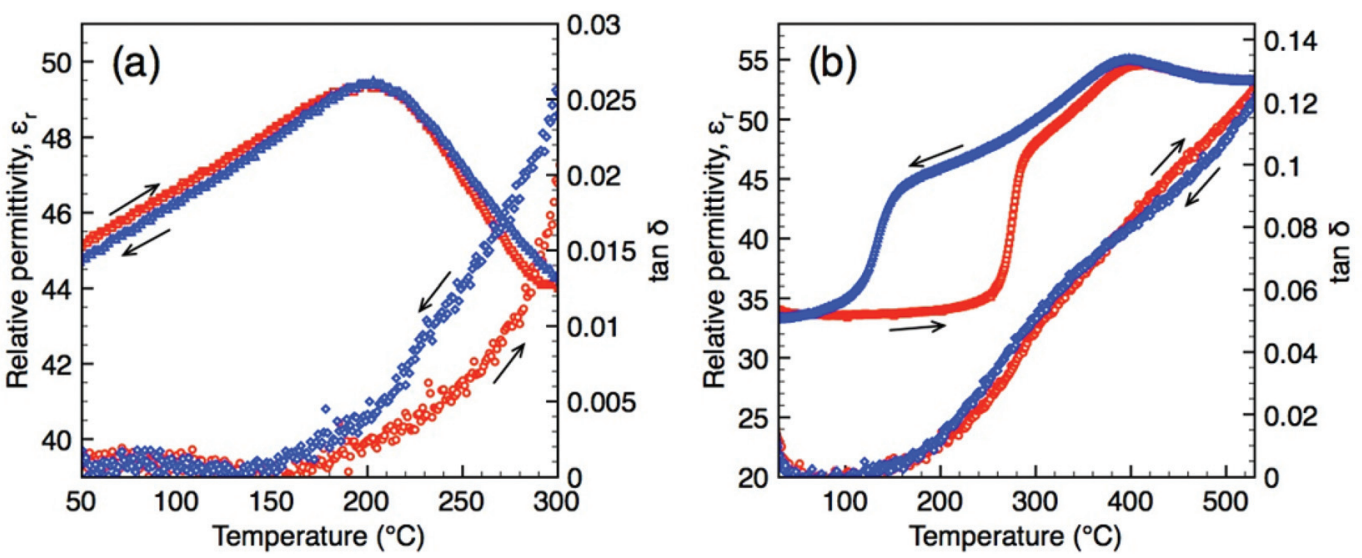

Fig. 8 Dielectric data (obtained at $1 \mathrm{MHz}$ ) as a function of temperature for $\mathrm{LaTaO}_{4}$ (a) and $\mathrm{La}_{0.90} \mathrm{Nd}_{0.10} \mathrm{TaO}_{4}$ (b).

samples became increasingly leaky (ESI $\dagger$ ) and so no conclusive evidence for ferroelectric switching was obtained. The polar (orthorhombic) phase of $\mathrm{La}_{0.9} \mathrm{Nd}_{0.1} \mathrm{TaO}_{4}$ is only stable at temperatures above our measuring capabilities.

\section{Structural comparisons}

Both structure types, $\mathrm{M}$ - and $\mathrm{O}-$, can be regarded as derived from an aristotype phase having no octahedral tilting, which may be described in space group $\mathrm{Cmcm}(a \sim 4 \AA, b \sim 14.8 \AA$, $c \sim 5.65 \AA(\sim a \sqrt{ } 2)$, as shown in Fig. 1(a). Naturally, there are many possible ways to lower the symmetry of the aristotype phase through structural distortions; those based on 'octahedral tilting' have been classified for the wider family of $\mathrm{A}_{n} \mathrm{~B}_{n} \mathrm{X}_{3 n+2}$ phases by Levin. ${ }^{21}$ In Levin's notation, the O-LaTaO ${ }_{4}$ structure corresponds to the $\mathbf{a}^{+}$tilt system and the $\mathrm{M}-\mathrm{LaTaO}_{4}$ structure corresponds to $\mathbf{a}^{-} \mathbf{b}$ (note that this notation is similar but distinct from that used in the Glazer system for conventional perovskites). The key difference is that in the O-phase tilts of successive octahedra along the $a$-axis are in-phase, whereas in the M-phase the corresponding tilts ( $c$-axis) are outof-phase, and tilting relative to the other aristotype axes is also permitted (Fig. 1). This aspect of the tilting, coupled with the layered nature of these structures, leads to the topological ferroelectric nature of the $\mathbf{a}^{+}$tilt system, but allows retention of centrosymmetricity in the $\mathbf{a}^{-}$tilt system.

Using first-principles calculations, Ederer and Spaldin $^{6}$ suggested that the topological ferroelectricity observed in the $\mathrm{BaMF}_{4}$ family was driven by a single polar soft-mode, essentially a 'rigid' octahedral tilt. However, employing similar methods, López-Pérez and Íñiguez ${ }^{7}$ found that in the $n=4$ member of this layered perovskite family, $\mathrm{La}_{2} \mathrm{Ti}_{2} \mathrm{O}_{7}$, the cooperative interaction of two distinct soft-modes is fundamentally important. They suggest that although the tilt mode is the driver for polarity, coupling with an octahedral distortion mode is significant, and is actually the majority contributor to the overall calculated polarisation. This may suggest that the conventional ' $\mathrm{d}{ }^{0}$-ness' criterion of ferroelectric activity does actually play a part in the oxide members of this family but not so much in the fluorides. In order to probe these effects further we can compare the degree of distortion within the octahedral units of several of these phases. Firstly, from the present work we can analyse the distortions present in the O-phase of $\mathrm{LaTaO}_{4}$ over a range of temperatures, and compare this to the $\mathrm{O}$ - and M-phases in $\mathrm{La}_{0.9} \mathrm{Nd}_{0.1} \mathrm{TaO}_{4}$. We can define simple 'distortion indices' for the octahedra, based on deviations of the six bond lengths from the mean, and the twelve bond angles from $90^{\circ}$ :

$$
\begin{aligned}
& \Delta_{1}=1 / 6 \sum\left|R_{\mathrm{av}}-R_{\mathrm{i}}\right| \\
& \Delta_{2}=1 / 12 \sum\left|90-\phi_{\mathrm{i}}\right|
\end{aligned}
$$

where $\phi_{\mathrm{i}}$ and $R_{\mathrm{i}}$ are the individual bond angles and bond lengths, $R_{\mathrm{av}}$ is the average bond length within the octahedron.

These are presented in Table 1, and compared to selected examples from the $\mathrm{BaMF}_{4}$ series, based on well-determined single crystal X-ray studies. ${ }^{22,23}$ Also included for comparison are values for the related $n=4$ phases $\mathrm{La}_{2} \mathrm{Ti}_{2} \mathrm{O}_{7}$ (theoretical study ${ }^{7}$ ) and $\mathrm{Sr}_{2} \mathrm{Nb}_{2} \mathrm{O}_{7}$ (single crystal X-ray study ${ }^{24}$ ), and for $\mathrm{BiReO}_{4}$ and $\mathrm{NaAlF}_{4}$, which both adopt the parent $\mathrm{Cmcm}$ structure for the $n=2$ series. $^{25,26}$ In the case of the $n=4$ phases there are two distinct B sites (Fig. 9), representing 'inner two' and 'outer two' octahedra of each perovskite block. It has previously been noted $^{8}$ that the distortion of the outer octahedra

Table 1 Comparison of octahedral distortions in selected oxide and

\begin{tabular}{|c|c|c|c|}
\hline Phase & $\Delta 1(\AA)$ & $\Delta 2\left(^{\circ}\right)$ & Distortion type \\
\hline $\mathrm{O}-\mathrm{LaTaO}_{4}\left(100^{\circ} \mathrm{C}\right)$ & 0.044 & 5.9 & Edge \\
\hline $\mathrm{O}-\mathrm{LaTaO}_{4}\left(500^{\circ} \mathrm{C}\right)$ & 0.073 & 5.0 & Edge \\
\hline $\mathrm{M}-\mathrm{La}_{0.9} \mathrm{Nd}_{0.1} \mathrm{TaO}_{4}\left(100^{\circ} \mathrm{C}\right)$ & 0.039 & 4.8 & Indistinct \\
\hline $\mathrm{O}-\mathrm{La}_{0.9} \mathrm{Nd}_{0.1} \mathrm{TaO}_{4}\left(500^{\circ} \mathrm{C}\right)$ & 0.053 & 5.1 & Edge \\
\hline $\mathrm{O}-\mathrm{BaMnF}_{4}\left(25^{\circ} \mathrm{C}\right)^{22}$ & 0.024 & 4.3 & Indistinct \\
\hline $\mathrm{O}-\mathrm{BaCoF}_{4}\left(25^{\circ} \mathrm{C}\right)^{22}$ & 0.047 & 3.3 & Axial \\
\hline $\mathrm{O}-\mathrm{BaNiF}_{4}\left(25^{\circ} \mathrm{C}\right)^{22}$ & 0.055 & 3.1 & Axial \\
\hline $\mathrm{M}-\mathrm{NaCrF}_{4}\left(25^{\circ} \mathrm{C}\right)^{23}$ & 0.031 & 2.0 & Edge \\
\hline $\mathrm{NaAlF}_{4}\left(25^{\circ} \mathrm{C}\right)^{25}$ & 0.018 & 1.3 & Edge \\
\hline $\mathrm{BiReO}_{4}\left(25^{\circ} \mathrm{C}\right)^{26}$ & 0.056 & 3.2 & Edge \\
\hline $\mathrm{O}-\mathrm{La}_{2} \mathrm{Ti}_{2} \mathrm{O}_{7}(\text { calc })^{7}$ & $0.088 / 0.071$ & $6.2 / 5.6$ & Apex \\
\hline $\mathrm{O}-\mathrm{Sr}_{2} \mathrm{Nb}_{2} \mathrm{O}_{7}\left(25^{\circ} \mathrm{C}\right)^{24}$ & $0.125 / 0.084$ & $7.3 / 5.6$ & Apex \\
\hline
\end{tabular}
fluoride members of the $A_{n} B_{n} X_{3 n+2}$ family 
(a)

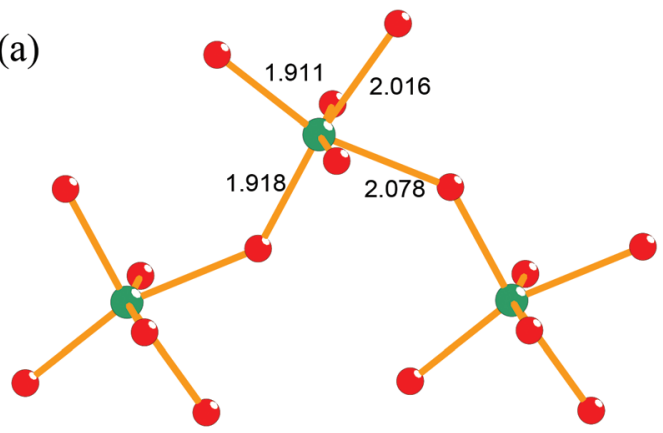

(b)

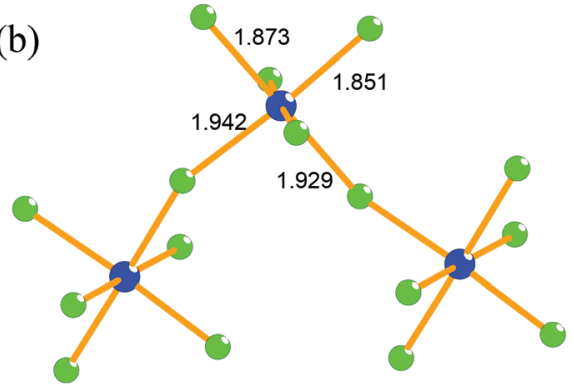

(c)

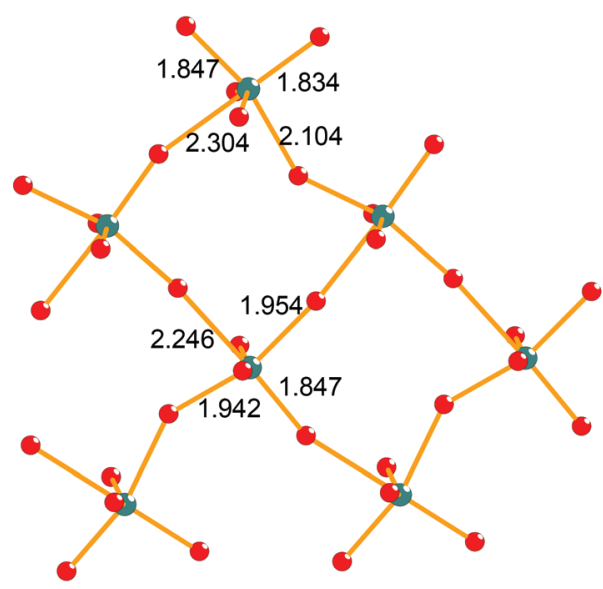

Fig. 9 Nature of the octahedral distortions in selected members of the $\mathrm{A}_{n} \mathrm{~B}_{n} \mathrm{X}_{3 n+2}$ family: (a) $\mathrm{O}-\mathrm{LaTaO}_{4}$ at $100{ }^{\circ} \mathrm{C}$ (present study) (b) $\mathrm{M}-\mathrm{NaCrF}_{4}$ at $25{ }^{\circ} \mathrm{C}$ (ref. 23) (c) $\mathrm{O}-\mathrm{Sr}_{2} \mathrm{Nb}_{2} \mathrm{O}_{7}$ at $25^{\circ} \mathrm{C}$ (ref. 24). Note that the latter contains two distinct octahedral sites.

are greater than those of the inner ones; this is a natural consequence of the weaker inter-block versus intra-block bonding (i.e., the inner octahedra are directly linked to six neighbouring octahedra, whereas the outer ones are linked to four).

As a further comparison to these values, the corresponding $\Delta 1$ and $\Delta 2$ values for the tetragonal ferroelectric phase of $\mathrm{BaTiO}_{3}$ at room temperature are $0.067 \AA$ and $2.5^{\circ}$, respectively. ${ }^{27}$

It can be immediately seen that all the examples given do have significant octahedral distortions. Moreover, these distortions have a significant component along the polar $c$-axis in the O-phases, and hence will contribute to any net polaris- ation. From the relatively limited data available it can also be suggested that (i) the oxide members of the $n=2$ series generally have larger distortions than the fluorides, (ii) the $n=$ 4 members of the series have enhanced octahedral distortions relative to the $n=2$ members, even in the case of the 'inner' octahedral layers. However, the representation of the octahedral distortions in terms of these two numerical parameters does not necessarily tell the full story; the nature of the distortion differs, as represented in Fig. 9. In general, the B cation in a perovskite can be considered to be displaced towards an octahedral apex, edge or face (compare the tetragonal, orthorhombic and rhombohedral ferroelectric phases of $\mathrm{BaTiO}_{3}$, for example). Also shown in Table 1, and illustrated for selected examples in Fig. 9, is a rough classification of the different distortion types present in this family. As can be seen, for the fluorides there is no clear trend to the distortion types, whereas for the oxides there are two distinct types of displacement: edge-oriented for the $n=2 \mathrm{O}$-phases and apex-oriented for the $n=4$ O-phases. Due to the limited data available, it is dangerous to draw any global conclusion from this, but it is clear that the $\mathrm{d}^{0}$ cations do display the types of distortion commonly seen in oxides; whether there is an inherent preference for a particular cation to show a particular type of distortion is unclear. Such effects have been discussed by Halasyamani and co-workers ${ }^{28}$ who suggest that $\mathrm{Ti}^{4+}, \mathrm{Nb}^{5+}$ and $\mathrm{Ta}^{5+}$ show roughly similar preferences for edge- and apex-distortions. Therefore it is likely that the specific details of inter-block versus intra-block bonding and the nature of the A cation in these complex, highly distorted structures is also significant.

We suggest that, although rigid octahedral tilting in the O-phases intrinsically provides a polarisation, in reality the octahedra are far from rigid, and the complex nature and differences in these deviations from ideality are not necessarily straightforward to predict, depending on a subtle difference in compatibility of the perovskite blocks and separating layers, together with the size and electronic nature of the B cations.

\section{Conclusions and further work}

We have studied various aspects of the phase behaviour of $\mathrm{LaTaO}_{4}$ and related members of the $\mathrm{A}_{n} \mathrm{~B}_{n} \mathrm{X}_{3 n+2}$ series of layered perovskites. This work demonstrates that, under appropriate reaction conditions, the polar $\mathrm{O}-\mathrm{LaTaO}_{4}$ phase can be stabilised at room temperature, whereas previous work had suggested that the centrosymmetric $\mathrm{M}-\mathrm{LaTaO}_{4}$ polymorph is dominant. Variable temperature powder diffraction studies reveal an unusual anisotropic thermal expansion effect, driven by anomalous behaviour of inter-octahedral bond angles. This structural anomaly correlates with a dielectric maximum. Despite the polar nature of this polymorph it has not been possible to demonstrate ferroelectric switching under the conditions employed here.

Further, we have shown that the $\mathrm{M}-\mathrm{LaTaO}_{4}$ polymorph can be stabilised to room temperature by a small amount of $\mathrm{Nd}$ doping at the La site: a consequence of the smaller A-cation 
size. In the series $\mathrm{La}_{0.9} \mathrm{Nd}_{0.1} \mathrm{TaO}_{4}$ we have shown that the stability range of the $\mathrm{M}$-phase at ambient temperature extends to $x \sim 0.50$. For the $x=0.1$ case, we have shown that a first-order phase transition from the $\mathbf{M}$ - to the O-phase occurs in the region $250-350{ }^{\circ} \mathrm{C}$. It is anticipated that the temperature of this phase transition will increase markedly for higher $x$-values, and further work on this is prompted. The dielectric data for $x=0.1$ also suggests a further structural feature to be present at $c a .400{ }^{\circ} \mathrm{C}$, and the intriguing possibility of antiferroelectric ordering; this also requires further study.

By comparing the present crystallographic data with selected previous examples of this structural family, we conclude that significant octahedral distortions are prevalent in all cases. This supports the previous theoretical studies, which suggest that although rigid octahedral tilting drives the transition into the polar O-phase, octahedral distortions are key in enhancing and stabilising the polarisation in this topological ferroelectric family. Although some broad general comments can be made on the nature and magnitude of these distortions, there is insufficient high quality structural data available to clarify specific 'design' principles, for the optimisation of net polarisation. The contributions of octahedral tilting versus octahedral distortion should therefore be considered on a case-by-case basis, and further systematic structural studies, on a more diverse range of compositions and structural variants, need to be carried out.

The transformation of an ambient temperature centrosymmetric phase into a higher temperature polar phase is highly unusual; indeed we are aware of no other examples of this amongst oxide ferroelectrics. It is anticipated that both the M- and O-polymorphs within this family will transform to an aristotype centrosymmetric phase (space group $\mathrm{Cmcm}$ ) at high temperature. Although examples of the aristotype phase have been structurally characterised (e.g., $\mathrm{BiReO}_{4}$ and $\mathrm{Sr}_{2} \mathrm{Ta}_{2} \mathrm{O}_{7}$, which transforms to the polar O-phase at $-107^{\circ} \mathrm{C}$ ), the nature of this transition has not been studied in detail previously. This would provide a very interesting study, in order to probe the relative importance of the octahedral tilt and distortion modes near $T_{\mathrm{C}}$.

\section{Note added in proof}

We note the recent observation of a uniaxial NTE in another family of layered perovskites (Senn et al., Phys. Rev. Lett., 2015, 114, 035701).

\section{Acknowledgements}

We thank the University of St Andrews and EPSRC (via DTG studentships to CALD and JG) for funding, STFC for providing neutron facilities and Cameron Black and Irene Munaò for experimental assistance. MS would like to acknowledge the International Association for the Exchange of Students for Technical Experience (IAESTE) for the opportunity to study at St Andrews.

\section{Notes and references}

1 R. E. Cohen, Nature, 1992, 358, 136.

2 B. B. van Aken, T. T. M. Palstra, A. Filippetti and N. A. Spaldin, Nat. Mater., 2004, 3, 164.

3 C. J. Fennie and K. M. Rabe, Phys. Rev. B: Condens. Matter, 2005, 72, 100103.

4 A. S. Gibbs, K. S. Knight and P. Lightfoot, Phys. Rev. B: Condens. Matter, 2011, 83, 094111.

5 N. A. Benedek and C. J. Fennie, Phys. Rev. Lett., 2011, 106, 107204.

6 C. Ederer and N. A. Spaldin, Phys. Rev. B: Condens. Matter, 2006, 74, 024102.

7 J. López-Pérez and J. Íñiguez, Phys. Rev. B: Condens. Matter, 2011, 84, 075121.

8 F. Lichtenberg, A. Herrnberger and K. Wiedenmann, Prog. Solid State Chem., 2008, 36, 253.

9 R. J. Cava and R. S. Roth, J. Solid State Chem., 1981, 36, 139.

10 F. Vullum, F. Nitsche, S. M. Selbach and T. Grande, J. Solid State Chem., 2008, 181, 2580.

11 I. Hartenbach, F. Lissner, T. Nikelski, S. F. Meier, H. MüllerBunz and T. Schleid, Z. Anorg. Allg. Chem., 2005, 631, 2377.

12 K. P. F. Siqueira and A. Dias, Mater. Res., 2014, 17, 167.

13 M. Nyman, M. A. Rodriguez, L. E. S. Rohwer, J. E. Martin, M. Waller and F. E. Osterloh, Chem. Mater., 2009, 21, 4731.

14 A. C. Larson and R. B. Von Dreele, Los Alamos National Laboratory Report No. LA-UR-86-748, 2000 (unpublished).

15 A. Wölfel, P. Dorscht, F. Lichtenberg and S. van Smaalen, Acta Crystallogr., Sect. B: Struct. Sci., 2013, 69, 137.

16 Yu. A. Titov, A. M. Sych, A. N. Sokolov, A. A. Kapshuk, V. Ya. Markiv and N. M. Belyavina, J. Alloys Compd., 2000, 311, 252.

17 Y. Gagou, Y. Amira, I. Lukyanchuk, D. Mezzane, M. Courty, C. Masquelier, Yu. I. Yuzyuk and M. El Marssi, J. Appl. Phys., 2014, 115, 064104.

18 M. Hidaka, T. Nakayama, J. F. Scott and J. S. Storey, Physica $B$ and $C, 1987,144,310$.

19 K. Shimamura, E. G. Víllora, H. Zeng, M. Nakamura, S. Takekawa and K. Kitamura, Appl. Phys. Lett., 2006, 89, 232911.

20 E. G. Víllora, K. Shimamura, F. Jing, A. Medvedev, S. Takekawa and K. Kitamura, Appl. Phys. Lett., 2007, 90, 192909.

21 I. Levin and L. A. Bendersky, Acta Crystallogr., Sect. B: Struct. Sci., 1999, 55, 853.

22 S. W. Kim, H. Y. Chang and P. S. Halasyamani, J. Am. Chem. Soc., 2010, 132, 17684.

23 G. Knoke, W. Verscharen and D. Babel, J. Chem. Res.Synop, 1979, 7, 213.

24 N. Ishizawa, F. Marumo, T. Kawamura and M. Kimura, Acta Crystallogr., Sect. B: Struct. Sci., 1975, 31, 1912.

25 A. Le Bail, Powder Diffr., 2009, 24, 301.

26 A. R. Rae-Smith and A. K. Cheetham, J. Solid State Chem., 1979, 30, 345.

27 G. H. Kwei, A. C. Lawson Jr., S. J. L. Billinge and S.-W. Cheong, J. Phys. Chem., 1993, 97, 2368.

28 K. M. Ok, P. S. Halasyamani, D. Casanova, M. Llunell, P. Alemany and S. Alvarez, Chem. Mater., 2006, 18, 3176. 